\title{
Electron Microscopy of Biological Macromolecules: Bridging the Gap Between What Physics Allows and What We Currently Can Get
}

\author{
Dieter Typke $^{1}$, Kenneth H. Downing ${ }^{1}$ and Robert M. Glaeser ${ }^{1,2 *}$
}

\author{
${ }^{1}$ Donner Laboratory, Lawrence Berkeley National Laboratory, University of California, \\ Berkeley, CA 94720, USA \\ 2 Department of Molecular and Cell Biology, University of California, Berkeley, CA \\ 94720-3206, USA
}

Key Words: Specimen motion, support films, image contract

Running title: IMPERFECT IMAGE QUALITY

* Corresponding author: Email: RMGlaeser@1bl.gov 
Abstract: The resolution achieved in low-dose electron microscopy of biological macromolecules is significantly worse than what can be obtained on the same microscopes with more robust specimens. When two-dimensional crystals are used, it is also apparent that the high-resolution image contrast is much less than what it could be if the images were perfect. Since specimen charging is one factor that might limit the contrast and resolution achieved with biological specimens, we have investigated the use of holey support films that have been coated with a metallic film before depositing specimens onto a thin carbon film that is suspended over the holes. Monolayer crystals of paraffin $\left(\mathrm{C}_{44} \mathrm{H}_{90}\right)$ are used as a test specimen for this work because of the relative ease in imaging Bragg spacings at $\sim 0.4 \mathrm{~nm}$ resolution, the relative ease of measuring the contrast in these images, and the similar degree of radiation sensitivity of these crystals when compared to biological macromolecules. A metallic coating on the surrounding support film does, indeed, produce a significant improvement in the high-resolution contrast for a small fraction of the images. The majority of images show little obvious improvement, however, and even the coated area of the support film continues to show a significant amount of beam-induced movement under low-dose conditions. The fact that the contrast in the best images can be as much as $25 \%-35 \%$ of what it would be in a perfect image is nevertheless encouraging, demonstrating that it should be possible, in principle, to achieve the same performance for every image. Routine data collection of this quality would make it possible to determine the structure of large, macromolecular complexes without the need to grow crystals of these difficult specimen materials. 


\section{INTRODUCTION}

The resolution that is currently achieved in electron microscopy of biological macromolecules lags far behind (1) the optical capabilities of modern instruments, (2) theoretical expectations of what should be physically possible to achieve, and (3) the resolution that is routinely achieved with most inorganic materials. It is natural to suppose that the high sensitivity of biological samples to radiation damage is responsible for the gap between what is physically possible and what is achieved in practice. The strict limitation on the amount of electron exposure that is tolerated by biological specimens does, indeed, result in extremely high levels of shot noise (statistical noise) from one high-resolution pixel to the next. This high level of shot noise does not account for the lag in image quality, however. The large gap in performance remains even when "safe" (i.e., low-dose) electron exposures are used to record images, and then spatial averaging of many identical molecules is used to overcome the shot-noise limitation. Although the image resolution achieved in this way can approach $0.3 \mathrm{~nm}$, the number of images that must be summed (averaged) is 100 times or more than what is theoretically required.

The fundamental problem in electron microscopy of biological macromolecules is the fact that the high-resolution image contrast is much lower than what scatteringphysics would allow it to be. Quantitative comparisons between the measured image contrast of two-dimensional crystals and the image contrast expected from electron diffraction patterns revealed that the image contrast in the best images was only $\sim 10 \%$ of what physical theory would allow it to be (Henderson and Glaeser, 1985), a situation that has not improved markedly with the use of the most modern types of electron microscope 
(Mitsuoka et al., 1999). Even worse, most images show no measurable signal at high resolution, even though one can be certain that the specimen itself always produces electron diffraction patterns with reproducible intensity. Both the magnitude of the highresolution image contrast that is obtained and the fraction of images that yield detectable contrast is improved by spot-scan imaging (Downing and Glaeser, 1986; Bullough and Henderson, 1987). This technique has yielded images of paraffin in which the best reported values of contrast were as high as $42 \%$ (Brink and Chiu, 1991) of what physical theory would allow. Even the use of spot-scan imaging does not come close to closing the gap between what physics allows and what the image contrast actually is for the majority of the images, however. This gap between actual image quality and what is theoretically possible is likely to be due to some type of beam-induced movement that occurs throughout the period of "safe" (low-dose) electron exposure.

It is natural to suggest that the observed beam-induced movement could consist of some type of image motion that is caused by electron-optical effects associated with specimen charging. On the other hand, semi-quantitative estimates of the amount of image deflection that might occur, for specimens prepared on ("conducting") carbon films, makes it seem more likely that the observed loss of contrast is due to some form of beam-induced physical movement of the specimen itself (Glaeser and Downing, 2003). This conclusion does not rule out the possibility, however, that charging can still occur within an insulating layer on the surface of the carbon film. Indeed, electrostatic stress associated with immobile surface charges may be one of the factors that cause beaminduced specimen movement. 
One remedy that is recommended as a way to improve image quality is to use carbon support films that have been pre-irradiated before applying sample materials to them (Miyazawa et al., 1999). The rationale in this case is that the conductivity of the carbon film is improved by pre-irradiation, and thus the limitations in image quality that are caused by specimen charging can be reduced by the use of pre-irradiated support films. The use of pre-irradiated carbon films has two unsatisfactory shortcomings. In the first instance, pre-irradiation introduces an inconvenient and time-consuming step in the process of sample preparation, one that the user would rather avoid if that were possible. Secondly, the improvement in conductivity produced by pre-irradiation is small. The purpose of the work that we report here has thus been to determine whether significant improvement in high-resolution image contrast might be achieved by using a metallic coating to improve the conductivity of evaporated carbon support films.

We have investigated the use of either gold or titanium/silicon (TiSi) alloy as conductive layers that can be evaporated onto holey carbon films before using the coated films for sample preparation. The use of a strongly scattering, metallic coating does not represent an acceptable solution when the specimen is deposited directly onto the support film, of course. On the other hand, there is little objection to using a metallic coating on the support film when the samples are actually prepared as vitreous ice films that are selfsupported within small holes. Since the use of holey carbon films has become a standard way to prepare biological specimens in electron cryo-microscopy (cryo-EM), the use of a metallic coating represents a reasonable alternative to pre-irradiated carbon films. The use of gold-coated holey carbon films had, in fact, been strongly recommended over 40 
years ago as a way to produce a very stable "microgrid", but that suggestion is not commonly adopted in current practice.

We have used uniformly thick crystals of paraffin as a convenient, "highresolution" test specimen for the current work. Paraffin has a sensitivity to radiation damage that is similar to that of biological macromolecules, and at the same time these crystals simplify the type of quantitative comparison that we wish to make between the experimentally achieved image contrast and that which would be present in a theoretically perfect image.

In the results reported here, we have found that the use of a conductive coating on holey carbon support films does, indeed, improve the high-resolution contrast that is recorded in the best $1-2 \%$ of the images. The contrast in the very best image has even exceeded $1 / 3$ of what is physically possible. Surprisingly, in view of this improvement in the quality at the top end of the data, the image contrast in the majority of images still remains extremely poor, with no qualitatively obvious improvement relative to that obtained with uncoated carbon support films. The suspicion that beam-induced specimen movement continues to be responsible for the poor image quality is confirmed by the observation of "chaotic" movement in low-dose images of the support film itself. Thus, while we can recommend the use of an evaporated metallic coating on the holey support films that are used in cryo-EM, other solutions are still required as well, in order to fully overcome the problem of beam-induced sample movement. 


\section{MATERIALS AND METHODS}

\section{Sample Preparation}

Carbon films containing randomly distributed, $1.5 \mu \mathrm{m}$ holes were prepared as 20 $40 \mathrm{~nm}$ thick replicas of Nuclepore filters (Whatman, Newton, MA). A parting layer of sodium metaphosphate (Victawet, SPI Supplies, West Chester, PA) was evaporated onto the Nuclepore filter before evaporating the carbon film. After transferring the holey carbon film to 400 mesh copper grids, these were either used as prepared or, alternatively, a thin layer (estimated at $<2 \mathrm{~nm}$ ) of either gold or titanium/silicon (TiSi) alloy (a gift from Dr. Michael Lamvik; see Rader and Lamvik, 1992) was evaporated onto one side of the carbon film. In either case, a thin carbon film (estimated at 5-10 nm) was then picked up on the holey support films in order to provide a continuous support film covering the holes.

Oriented, monolayer crystals of paraffin $\left(\mathrm{C}_{44} \mathrm{H}_{90}\right.$, Sigma-Aldrich) were grown on the continuous support films by solvent evaporation. Suitable specimens can be prepared by application of $\sim 3 \mu 1$ of a $\sim 1 / 3$-saturated solution of paraffin in hexane to a grid, which rapidly spreads and evaporates. This technique produces a variety of thin crystals that are up to several micrometers in area, distributed randomly over the surface of the continuous carbon film. Many of these crystals are diamond-shaped and uniformly thick (corresponding to the chain length of one molecule), while others are one molecule in thickness only over the thin border of a larger crystal. Each such grid thus contains numerous instances of areas that can be used for data collection, in which a significant portion of a single, monolayer crystal covers part or all of a hole in the holey carbon film. 


\section{Data Collection and Analysis}

All electron microscopy was carried out with a liquid nitrogen cooled specimen stage (Gatan, Pleasanton, California) on a $400 \mathrm{keV}$ electron microscope (JEOL 4000) equipped with $\mathrm{L} \mathrm{LaB}_{6}$ electron gun. Specimens were scanned with negligible amounts of pre-exposure by viewing the image formed by the central spot in a highly overfocused diffraction pattern, and promising crystals were confirmed to diffract as expected by briefly viewing the focused diffraction pattern. After locating a suitable specimen area, the objective lens defocus was set to a value of $\sim 300 \mathrm{~nm}$ in an area $\sim 2.5 \mu \mathrm{m}$ to one side of the area from which data were to be collected. High-resolution images were recorded on photographic film at a nominal magnification of 60,000 . An electron exposure of $1500-2500$ electron $/ \mathrm{nm}^{2}$ was used to record images, with the beam diameter adjusted to be slightly larger than (and symmetrically distributed around) the edge of the hole in the holey carbon film (Miyazawa et al., 1999).

Photographic films were digitized with a Nikon SuperCoolScan 8000 ED film scanner, using a home-modified film holder to accommodate the large $(3.25 \times 4$ inch) size of conventional EM negatives. Areas consisting of 8,964x11,016 pixels were digitized at a pixel size of $6.35 \mu \mathrm{m}$. The resulting values of percent transmission were converted to optical density, which in turn is proportional to the electron image intensity at the relatively low densities produced under the experimental conditions described above.

The Fourier transform of the digitized images was used to calculate the ratio of $\mathrm{F}_{\text {image }}(\mathrm{g})$, the amplitude of a Fourier component of the image intensity with a spatial frequency " $\mathrm{g}$ ", to $\mathrm{F}_{\text {image }}(0)$, the average value of the image intensity, which is due to the unscattered electron beam. This ratio, designated $F(g) / F(0)$ by Henderson and Glaeser 
(1985), is then used to compare the measured contrast to the highest value that it could theoretically be, based on the value of the corresponding ratio that is measured in an electron diffraction pattern (see the next paragraph for details). The values of $F_{\text {image }}(g)$ were calculated as the square root of the intensity integrated over an area of $5 \times 5$ pixels surrounding the peaks in the computed power spectrum. No background subtraction was needed in this case because the spot amplitudes were much higher than the background in the computed power spectra (see an example in Figure 1a). The $F_{\text {image }}(g) / F_{\text {image }}(0)$ values were then corrected for the combined modulation transfer function (MTF) of the film and the densitometer. The MTF was itself estimated from the power spectrum of pure shot noise that was recorded on an image of a hole in the specimen where there was no specimen or carbon film. Due to aliasing, the estimate of the MTF obtained in this way at half the Nyquist frequency, i.e. at the position of the high-resolution diffraction peaks of interest, is only slightly larger than its true value. The values that we report for the corrected $F_{\text {image }}(\mathrm{g}) / \mathrm{F}_{\text {image }}(0)$ ratios thus are actually slightly less than their true values.

Values of $F_{\text {diffraction }}(\mathrm{g}) / \mathrm{F}_{\text {diffraction }}(0)$ in the electron wave transmitted through the sample were measured as the square root of the ratio of electron diffraction intensities, $\mathrm{I}(\mathrm{g}) / \mathrm{I}(0)$. As in Henderson and Glaeser (1985), the intensity of the diffracted beam, I(g), and that of the unscattered beam, $\mathrm{I}(0)$, were measured by recording images of the defocused diffraction pattern. In this case the diffraction intensities were recorded with the Gatan 2kx2k CCD camera mounted on our JEOL 4000. As is shown in Figure 1b, the diffraction spots are defocused so as to form miniature dark field images, across which the intensity in each Bragg spot varies due to local bending of the crystal. The intensity across the central spot, recorded with a much lower exposure than that shown in Figure 
$1 b$, is effectively constant, however, since the specimen is very thin. The brightest areas within each of the dark field images was used in order to estimate the maximum value of $\mathrm{I}(\mathrm{g}) / \mathrm{I}(0)$ that can be expected for the paraffin crystals, and this corresponds to a value of $\mathrm{F}_{\text {diffraction }}(\mathrm{g}) / \mathrm{F}_{\text {diffraction }}(0)=\sim 0.1$ at $400 \mathrm{keV}$. Referring to the analysis of Henderson and Glaeser (1985), the Fourier transform of a perfect image of such an area of the specimen would, in turn, produce a value of $\mathrm{F}_{\text {image }}(\mathrm{g}) / \mathrm{F}_{\text {image }}(0)=\sim 0.2$. The actual values of $F_{\text {image }}(g) / F_{\text {image }}(0)$ that are found in the best areas of such images, divided by 0.2 , thus provides a quantitative measure of the image quality. A value of $\sim 1$ for this ratio would correspond to a "perfect" image.

\section{RESULTS}

Images of specimens prepared with coated and uncoated holey support films were first evaluated by optical diffraction. Between $\sim 50$ and 100 images were recorded for each type of sample in order to make a reasonable statistical comparison of the results obtained with various types of support films. The computed power spectrum of a paraffin crystal that is shown in Figure 1a is from an exceptionally good image, in the sense that the six diffraction spots at Bragg spacings of 0.38 to $0.42 \mathrm{~nm}$ resolution are about equally intense, and even a relatively strong spot can be seen at a resolution of $0.25 \mathrm{~nm}$. The image used to compute this diffraction pattern was recorded from a specimen prepared on a TiSi-coated holey support film. The $0.25 \mathrm{~nm}$ diffraction spots could be detected in about $5-10 \%$ of the images when either gold-coated or TiSi-coated holey support films were used, whereas this resolution was not achieved in any of the images recorded with uncoated holey carbon films. 
Although the majority of micrographs contained some areas for which one could observe all three pairs of Bragg spots at a resolution of $\sim 0.4 \mathrm{~nm}$, many sub-areas of the digitized images contained only one or two pairs of diffraction spots. The asymmetry of the power spectra could be due to bending of the crystal, which we know gives rise to an asymmetric electron diffraction pattern to begin with (see Figure 1b), or it could be due to beam-induced movement that has occurred with a preferred direction (see below). In view of this uncertainty, we have limited our analysis of the measured $F_{\text {image }}(g) / F_{\text {image }}(0)$ ratio to the strongest diffraction spots recorded in any one image, ignoring the question of whether specimen tilt or beam-induced movement is responsible for the frequent absence of one or even two of the remaining pairs of diffraction spots.

Only the images that showed the most intense optical diffraction spots were then digitized. Power spectra were calculated for sub-areas 1024 pixels on edge, to identify the region of the photographic film that produced the strongest diffraction spots. The values of $F_{\text {image }}(g) / F_{\text {image }}(0)$ for the best areas were then corrected for the combined MTF of the photographic film and the densitometer, and these values were divided by 0.2 in order to estimate how strong the high-resolution image contrast was relative to what it could be. Table 1 lists the values obtained for the two best examples of specimens prepared on uncoated carbon holey films, Au-coated holey films, and TiSi-coated holey films, respectively.

The best images of paraffin crystals were those obtained with samples prepared on TiSi-coated holey films. The best of such images have $25 \%$ to $35 \%$ of the contrast that would be present in a perfect image. In the present experiments, however, as in the work published by Henderson and Glaeser (1985), which was conducted at $100 \mathrm{keV}$, the best 
$\mathrm{F}_{\text {image }}(\mathrm{g}) / \mathrm{F}_{\text {image }}(0)$ ratio that was obtained for samples prepared on uncoated carbon films was only $\sim 10 \%$ of what it would be in a perfect image. The contrast in the best images of specimens prepared on Au-coated holey support films shows only a slight improvement over that of specimens prepared on uncoated support films. We can add, however, that even better results have been obtained with Au-coated support films in preliminary experiments performed at helium temperature (results not shown).

The majority of images obtained with either Au-coated holey films or TiSi-coated holey films are, unfortunately, not qualitatively better in the resolution range of $\sim 4 \mathrm{~nm}$ than are those obtained with uncoated holey films. We have not made a quantitative comparison of the $\mathrm{F}_{\text {image }}(\mathrm{g}) / \mathrm{F}_{\text {image }}(0)$ ratio for all such images because of the large amount of effort that this would entail. Judging only on the basis of the apparent strength of the optical diffraction patterns, and on the basis of the number of diffraction spots visible in a single-crystal optical diffraction pattern, there is no obvious difference in the image quality for the different types of specimen. This is in contrast to the case of the few images that, for each type of specimen, give the strongest (best) optical diffraction patterns - in this case it was already apparent that the images obtained with TiSi-coated holey films were markedly better than those obtained with specimens prepared on uncoated holey films. Furthermore, as was mentioned above, the use of either Au-coated or TiSi-coated support films does increase the likelihood that diffraction spots will be present at $\sim 0.25 \mathrm{~nm}$ resolution.

Strongly defocused, low-dose images of the coated area of the support film provide clear evidence that beam-induced movement still occurs in a highly variable, i.e. unpredictable way over the illuminated area of a specimen. Figure 2 shows a 
representative example of a gallery of power spectra that were computed for different subareas of an image of a TiSi-coated support film, all recorded on the same micrograph. Each sub-area consisted of $512 \times 512$ pixels, and the center-to-center distance between adjacent sub-areas was $\sim 0.5 \mu \mathrm{m}$. The Nyquist frequency in these spectra is close to 2.4 $\mathrm{nm}^{-1}$, and indeed the $0.42 \mathrm{~nm}$ Bragg reflection from a paraffin crystal can be seen in some of the panels. The amount of specimen movement that has occurred in each sub-region of the image, and even the direction of the movement, varies quite substantially over distances as little as $\sim 0.5 \mu \mathrm{m}$, as is evident from the asymmetry of the Thon rings in some of the power spectra. The fact that the movement varies in amount and direction over the illuminated area rules out stage drift as the cause of the movement. The most asymmetric of the Thon rings furthermore appear to be underlaid by a weak, symmetrical power spectrum with the same radius, implying that the area in question may not have moved during some portion of the electron exposure. After an exposure of $\sim 5000$ electrons $/ \mathrm{nm}^{2}$ the observed, spatially variable specimen movement ceases (data not shown), and all areas then produce fully symmetric power spectra. This same type of spatially variable, beam-induced movement has long been noted by others, but it has only rarely (Hayward and Glaeser, 1980) been described in published work. 


\section{DISCUSSION}

The occasional improvement in image quality that has been obtained by using TiSi-coated holey support films represents welcome progress in the direction of closing the gap that exists between the quality of images that are normally obtained with biological specimens and what physics would allow the image quality to be. Major improvements are still needed in the consistency with which such images are obtained, however. The goal must still be for most, if not all, images to have the high quality that currently is shown by only the best images that are represented in Table 1 .

We find it difficult to imagine that the low-dose movement effects documented in Figure 2 could be the result of electron-optical effects that occur due to specimen charging. Points that make it unlikely that electron optical effects cause such localized movements include the fact that the specimen area contains a highly conducting layer. As a result, charging can only occur within an insulating surface layer, and the resulting electrostatic field will be that of a dipole-sheet (Glaeser and Downing, 2003). Furthermore, the specimen is essentially untilted, thereby providing little opportunity for the dipole layer to act as a deflecting prism (Glaeser and Downing, 2003). Localized electron-optical deflections that vary in direction and magnitude over the scale of less than a micrometer are difficult to rationalize under these circumstances.

We thus suggest that the observed low-dose movements are due, instead, to some form of beam-induced movement of the specimen. The mechanical stresses driving those movements may be due to the progressive accumulation of radiation damage in the beamsensitive specimen itself. Similar beam-induced stress might also be generated within a thin carbon film on which the sample is prepared, or which might be evaporated onto a 
frozen-hydrated specimen after preparation (Jakubowski et al., 1988). Alternatively, the accumulation of positive charge within a non-conducting surface layer, although compensated by freely mobile counter charges that accumulate within the conducting layer, may also generate large stresses through frustrated Coulombic repulsion (Glaeser and Downing, 2003). It is not sensible to "pre-irradiate" the specimen nor, in this case, the supporting carbon film, before collecting data of course. Although it is reasonable to suppose that the irradiated area of the specimen would reach some stable end point, after which further specimen motion would stop, the kinetics of the process correspond to electron exposures that completely damage the high-resolution features of biological specimens.

A few directions for further work suggest themselves. The use of much thicker conducting layers (which are unlikely to experience radiation-induced mechanical stress) may add further mechanical strength that can better resist the stresses that are unavoidably generated in the biological sample itself. Along this line, if robust, holey "microgrids" can be fabricated which themselves do not exhibit beam-induced movement under low-dose conditions, then it would seem advisable to support the biological specimens in (or over) holes of the smallest possible diameter so as to best take advantage of the mechanical support provided by the holey film.

Another alternative, mentioned already by Henderson and Glaeser (1985), would be to record images in a series of extremely short ("stroboscopic") exposures, which might be as little as $1 \%$ or less of the "safe" dose as far as radiation damage is concerned. The rationale in this approach is that the amount of beam-induced movement that can occur during a given period of exposure must decrease with the amount of exposure, and 
at some point the amount of movement per frame must become negligible. The lack of a suitable detector has so far been the primary barrier to implementation of this alternative. Stroboscopic image-capture fails when the signal associated with the electron exposure per frame is much less than the additive noise derived from the detector and the data readout. Photographic image capture is certainly not a possibility, for example, because the electron signal falls well below the fog level of film when images are recorded with significantly less exposure than is needed for the full, low-dose exposure. Improvement in CCD cameras or similar image-readout detectors, which can have a much lower level of detector noise than photographic film, may make it possible to investigate whether the concept of stroboscopic image capture represents a viable solution.

Realizing the benefit of stroboscopic imaging will also require that there be sufficient signal in each of the frames to allow them to all be computationally aligned, thereby correcting for the progressive movement that occurs in the course of the continuous exposure. If the alignment can be done for areas much larger than the size of a single particle, the exposures could be fractionated to an extent proportional to the area that would be used for alignment. In this regard it will be important to increase, as much as possible, the size of the field for which images are recorded with a CCD (or similar) detector. We note, however, that the currently observed, beam-induced motion is often not uniform over areas larger than a few hundred nanometers (see Figure 2, for example), and thus it would be futile to apply the alignment of stroboscopic images to areas larger than that.

The motivation for continuing to find ways to improve the quality of images of biological specimens has been well described (Henderson, 1995; Glaeser, 1999). To 
summarize, high-resolution structures could be produced from a large set of EM images of single macromolecules without requiring crystallization of the specimen, provided that there is sufficient signal in each such image to determine the position and orientation of each such molecule. If images were perfect, such work would be possible for proteins as small as $40,000 \mathrm{D}$, and the data sets would need to include fewer than 15,000 particles. Even if the images were not perfect but could be routinely collected with a signal of at least $35 \%$ of what physics would allow, high-resolution structures could still be obtained for particles in the size range of $400,000 \mathrm{D}$, and the required data sets would consist of $\sim 100,000$ particles. Particles of molecular weight $\sim 400 \mathrm{kD}$ encompass a large fraction of the macromolecular complexes and machines that are currently of great interest in biochemistry and cell biology, and the needed quantity of data could still be collected with quite high throughput. As a result, there is considerable justification to continue the search for methods to obtain images of biological specimens whose quality is routinely as high as has been realized in the top $1-2 \%$ of work reported here.

\section{ACKNOWLEDGEMENTS}

This work has been supported by NIH grant R01 GM 62989. 


\section{REFERENCES}

Brink, J. \& Chiu, W. (1991). Contrast analysis of cryo-images of n-paraffin recorded at $400 \mathrm{kV}$ out to $2.1 \AA$ resolution. J. Microscopy 161, 279-295.

Bullough, P. \& Henderson, R. (1987). Use of spot-scan procedure for recording low-dose micrographs of beam-sensitive specimens. Ultramicroscopy 21, 223-230.

Downing, K.H. \& Glaeser, R.M. (1986). Improvement in high resolution image quality of radiation-sensitive specimens achieved with reduced spot size of the electron beam. Ultramicroscopy 20, 269-278.

Glaeser, R.M. (1999). Review: Electron crystallography: present excitement, a nod to the past, anticipating the future. J Struct Biol 128, 3-14.

Glaeser, R.M. \& Downing, K.H. (2003). Specimen charging on thin films with one conducting layer: discussion of physical principles. Micros Microanal Submitted for publication.

Hayward, S.B. \& Glaeser, R.M. (1980). Use of low temperatures for electron diffraction and imaging of biological macromolecular arrays. In Electron Microscopy at Molecular Dimensions, Baumeister, W. \& Vogell, W. (Eds). pp. 226-233. Berlin: Springer-Verlag. 
Henderson, R. (1995). The potential and limitations of neutrons, electrons and x-rays for atomic resolution microscopy of unstained biological molecules. $Q$ Rev Biophys 28, 171193.

Henderson, R. \& Glaeser, R.M. (1985). Quantitative analysis of image contrast in electron micrographs of beam-sensitive crystals. Ultramicroscopy 16,139-150.

Jakubowski, U., Baumeister, W. \& Glaeser, R.M. (1989). Evaporated carbon stabilizes thin, frozen-hydrated specimens. Ultramicroscopy 31, 351-356.

Mitsuoka, K., Hirai, T., Murata, K., Miyazawa, A., Kidera, A., Kimura, Y. \& Fujiyoshi, Y. (1999). The structure of bacteriorhodopsin at $3.0 \AA$ resolution based on electron crystallography: implication of the charge distribution. J Mol Biol 286, 861-882.

Miyazawa, A., Fujiyoshi, Y., Stowell, M. \& Unwin, N. (1999). Nicotinic acetylcholine receptor at $4.6 \AA$ resolution: transverse tunnels in the channel wall. $J$ Mol Biol 288, 765786.

Rader, S.S. \& Lamvik, M.K. (1992). High-conductivity amorphous TiSi substrates for low-temperature electron microscopy. J Microscopy 168, 71-77. 
TABLE 1 Quantitative evaluation of the image contrast for the two best images identified within sets of 50-100 photographic negatives that were recorded for each type of specimen. Specimens were prepared on holey carbon films either with or without coating the carbon film with a metallic layer, as described in the section on Materials and Methods. The amplitude of the Fourier transform of the digitized image was determined for diffraction spots at a resolution of $\sim 0.4 \mathrm{~nm}$, and this amplitude was divided by the amplitude of the Fourier transform at zero frequency. The resulting ratio, $F_{\text {image }}(\mathrm{g}) / \mathrm{F}_{\text {image }}(0)$, is thus independent of the "speed" of the photographic film. In separate experiments the corresponding ratio of $F(g) / F(0)$ for electron diffraction patterns was determined to be $\sim 0.1$ for $400 \mathrm{keV}$ electrons, and thus $\mathrm{F}_{\text {image }}(\mathrm{g}) / \mathrm{F}_{\text {image }}(0)$ would be 0.2 for a theoretically perfect image of a "weak phase" object. The ratio of values of $\mathrm{F}(\mathrm{g}) / \mathrm{F}(0)$ that are shown in the right hand column of the table are the values computed from the digitized images, divided by 0.2 . If the experimental images were perfect, the resulting value would be 1.0. The very best image obtained in this series of micrographs is therefore better than $1 / 3$ of what physics would permit. 


\begin{tabular}{c|c} 
Type of holey support film & $\frac{F_{\text {image }}(\mathrm{g}) / \mathrm{F}_{\text {image }}(0)}{2 \mathrm{~F}_{\text {diffraction }}(\mathrm{g}) / \mathrm{F}_{\text {diffraction }}(0)}$ \\
\hline Carbon only & $0.07,0.09$ \\
\hline Gold-coated carbon & $0.11,0.14$ \\
\hline TiSi-coated carbon & $0.23,0.36$ \\
\hline
\end{tabular}




\section{FIGURE LEGENDS}

Figure 1 Diffraction patterns obtained with the monolayer crystals of paraffin that were used as a test specimen. (A) Diffraction pattern (power spectrum) computed for a digitized image that was recorded on photographic film. The specimen was prepared on a continuous carbon film, supported by a holey carbon film that had been coated with a metallic, amorphous film of titanium/silicon (TiSi) alloy (Rader and Lamvik, 1992). This example is an unusually "good" example in which all 3 pairs of diffraction spots at a resolution of $\sim 0.4 \mathrm{~nm}$ are almost equally strong, and even the diffraction spots at $\sim 0.25$ $\mathrm{nm}$ resolution are present. (B) Defocused electron diffraction pattern recorded from an illuminated area that is $\sim 2 \mu \mathrm{m}$ in diameter. The intermediate (diffraction) lens was intentionally overfocused in order to produce this image, in which each diffraction spot represents a miniature "dark field" image of the illuminated area of the specimen. Wrinkling of the thin crystal causes bend contours, i.e. regions within which the crystal either is or is not bent so as to satisfy the Bragg condition for that particular diffraction spot. The resolution of the quasi-hexagonal spots is $\sim 0.4 \mathrm{~nm}$, while the pair of diffraction spots at higher resolution corresponds to a Bragg spacing of $\sim 0.25 \mathrm{~nm}$. 
Figure 2 Power spectra computed for adjacent areas of a highly defocused image of TiSicoated carbon, recorded on a single photographic film. Each spectrum shows the Fourier transform of an area that is 256 pixels (corresponding to $55 \mathrm{~nm}$ ) on edge, and the centerto-center distance between the adjacent areas is only $500 \mathrm{~nm}$. Although there is some astigmatism in these images, the dominant reason for asymmetry is specimen movement, which varies in magnitude and direction over much of the recorded image. The first Thon ring occurs at a resolution of $\sim 1.8 \mathrm{~nm}$, and diffraction spots from a paraffin crystal are visible at nearly the Nyquist frequency in some of the panels. The power spectrum in the lower left panel shows clear evidence for some signal in the "bad" direction of the first Thon ring, suggesting that the motion may have been less severe during part of the full period of electron exposure. The spectra in the top right and lower right panels are weaker than the others because the incident beam intensity in these areas was not as intense as in the rest of the field of illumination. 


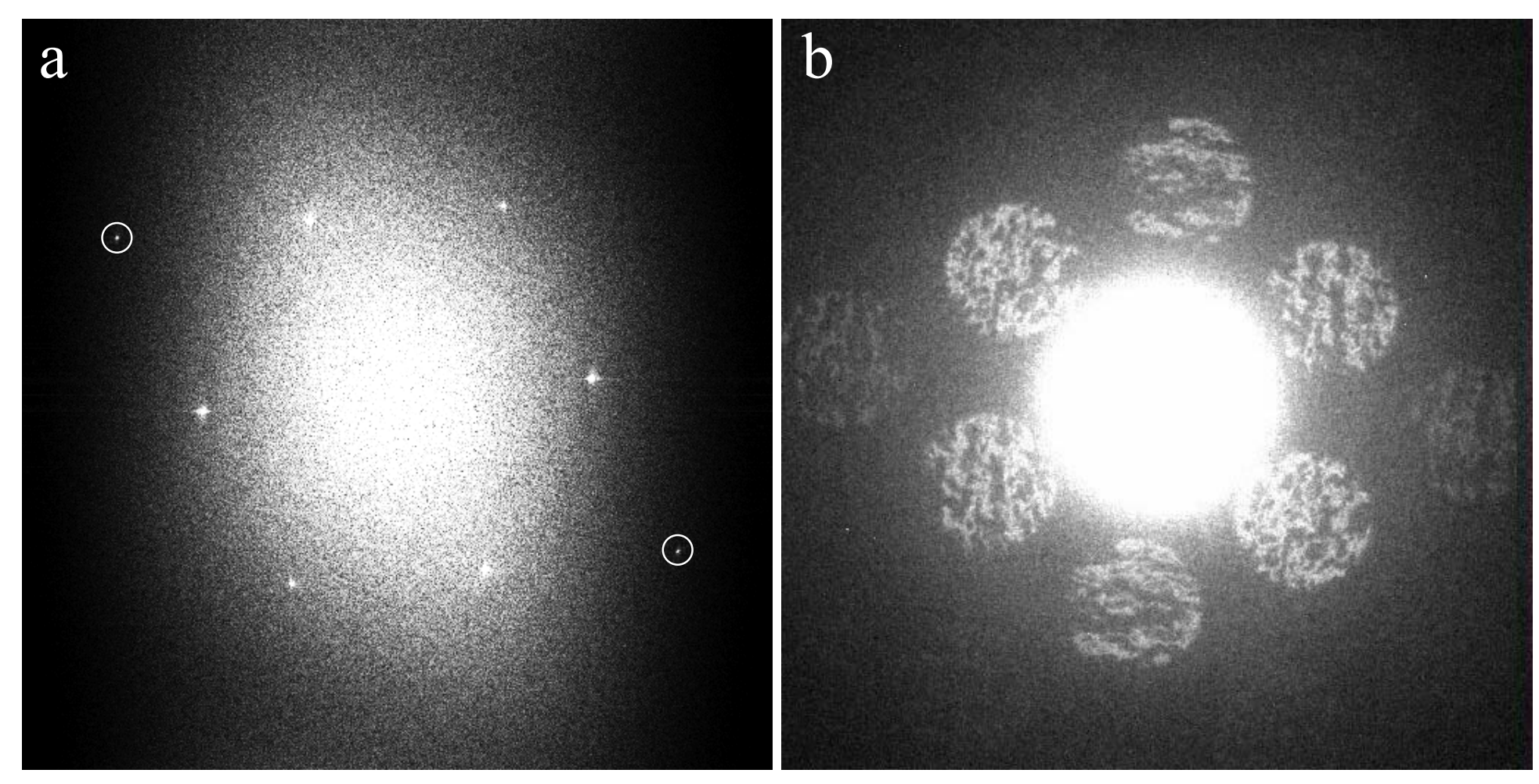




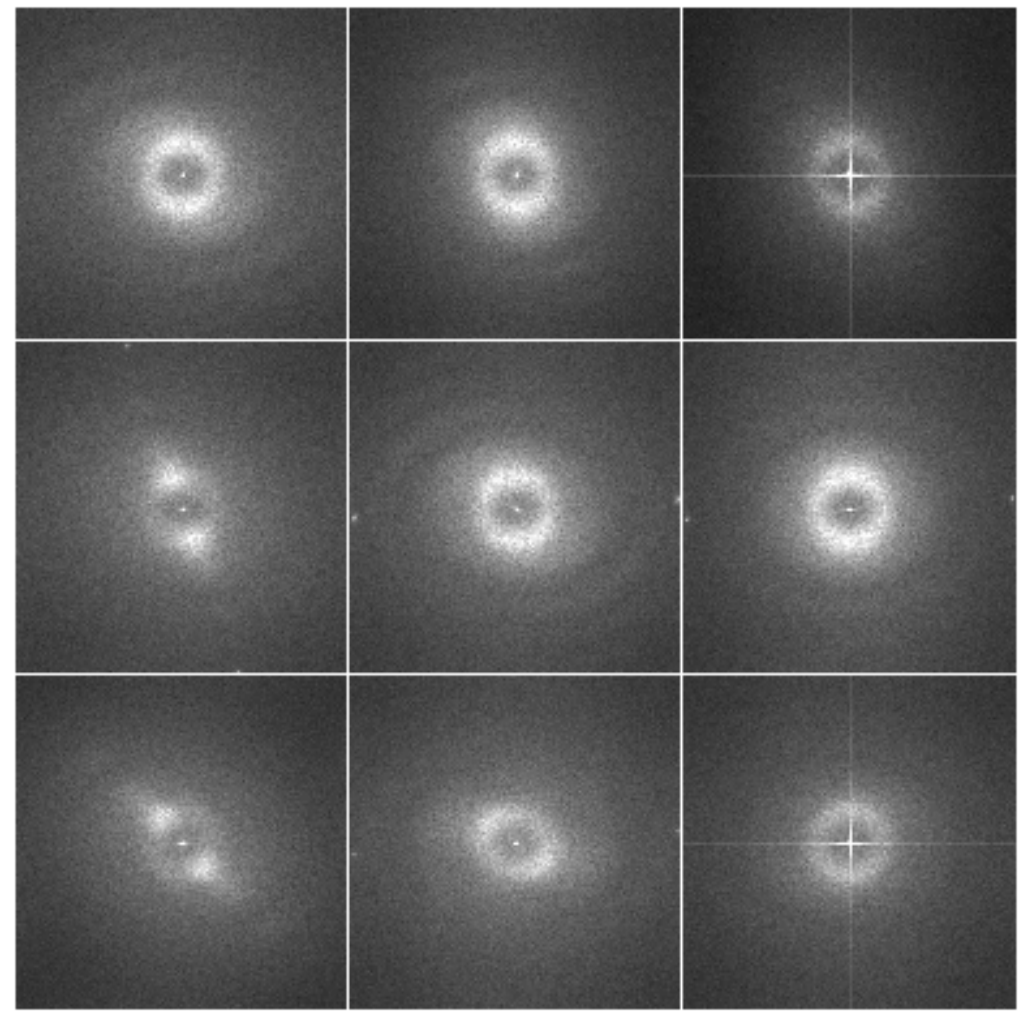

